\title{
Rydberg states of hydrogenlike ions in a braneworld model
}

\author{
F. Dahia ${ }^{a}$, E. Maciel, A. S. Lemos \\ Department of Physics, Federal University of Paraíba, João Pessoa, PB, Brazil
}

Received: 6 May 2018 / Accepted: 15 June 2018 / Published online: 27 June 2018

(C) The Author(s) 2018

\begin{abstract}
Precise measurements of optical transition frequencies between Rydberg states of hydrogen-like ions could be used to obtain an improved value of the Rydberg constant, by avoiding the uncertainties about the proton radius. Motivated by this perspective, we investigate the influence of the gravitational interaction on the energy levels of Hydrogenlike ions in Rydberg states in a braneworld model. As it is known, in this scenario, the gravitational interaction is amplified in short distances. We show that, for Rydberg states, the main contribution for the gravitational potential energy does not come from the rest energy concentrated on the nucleus but from the energy of the electromagnetic field created by its electric charge. The reason is connected to the fact that, when the ion is in a Rydberg state with high angular momentum, the gravitational potential is not computable in zero-width brane approximation due to the gravitational influence of the electrovacuum in which the lepton is moving. Considering a thick brane scenario, we calculate the gravitational potential energy associated to the nucleus charge in terms of the confinement parameter of the electric field in the brane. We show that the gravitational effects on the energy levels of a Rydberg state can be amplified by hidden dimensions even when the compactification scale is shorter than the Bohr radius.
\end{abstract}

\section{Introduction}

In the past decades we have seen a renewed interest in extradimensional theories stimulated by the braneworld scenario, according to which our ordinary universe is a submanifold embedded in a higher-dimensional space [1-4]. The fundamental characteristic of these models is the assumption that matter and fields are confined in a three-dimensional space (the 3-brane), while gravity has access to the whole space, implying that the gravitational field could feel direct effects of the extra dimensions on a length scale much greater than the

a e-mail: fdahia@ fisica.ufpb.br scale where the standard model fields are directly affected. Since the gravitational interaction has been tested in short distance only recently, then braneworld scenario may allow the formulation of phenomenologically viable models in which the hidden dimensions can be much larger than the fourdimensional Planck length $\left(10^{-35} \mathrm{~m}\right)$, which is the characteristic size of the fifth direction postulated by the Kaluza-Klein model, the first modern higher-dimensional theory.

Direct laboratory tests of the inverse square law impose that the compactification radius $(R)$ should be less than $44 \mu \mathrm{m}[5,6]$, for instance. This is the most stringent constraint when the supplementary space has only one extra dimension. Regarding greater codimensions, experimental limits from astrophysics and colliders are tighter, but, according to current data, they are still greater than Planck length by 15 orders at least [7-14].

The original purpose of braneworld theories was to explain the hierarchy problem, i.e., why gravity is so much weaker in comparison to the other fundamental forces [1-4]. According to these models, the reason, roughly speaking, is that gravity is the only field that spreads in every direction, hence it seems weaker than the other interactions in the long distance scale, where the usual four-dimensional behavior is recovered.

On the other hand, in distances smaller than the size of the extra dimensions, these models predict that the gravitational force is amplified in comparison to the Newtonian three-dimensional force by a factor that depends on the number and on the size of the additional dimensions. This fact has motivated many authors to study the effects of the gravitational field in the atomic and molecular system as a way to obtain independent experimental bounds for parameters of the higher-dimensional theories [15-22].

Here we intend to study the influence of the extra dimensions on Rydberg states of hydrogen-like ions, motivated by recent developments in the spectroscopy area which suggest that precision measurements of optical transitions between Rydberg states can become an efficient method to test QED 
theory and also to determine the value of the Rydberg constant more accurately [23].

The current uncertainty of Rydberg constant is of the order of $10^{-12}$ [24] and its value is extracted from the comparison of theoretical predictions and measurements of transition frequencies in hydrogen and deuteron, which includes transitions between $S$-states [23,24]. It happens that, regarding $S$-states, theoretical calculations are mainly limited by the uncertainty about the proton charge radius [23], whose value is also a source of a controversy known as the proton radius puzzle [27-30], which is a discrepancy between measurements of the proton size as inferred from the muonic hydrogen spectroscopy and the CODATA value [24], based on the proton-electron interaction.

In Rydberg states with high angular momentum, as the influence of the internal structure of the nucleus is negligible, the predictions are practically independent of the proton radius and then measurements would be free from those uncertainties. With recent calculations of QED corrections for higher-quantum number states, the accuracy of the predicted energy level could reach parts in $10^{17}$ [23]. At the same time, advances on experimental techniques, such as optical frequency combs [25], promises to reduce the relative uncertainties to the order of $10^{-19}$ in measurements of transition frequencies around the optical range [26]. Together, these theoretical and experimental advances may allow precise measurements of certain optical transitions between appropriate Rydberg states which, in principle, could lead to an improved value of the Rydberg constant [23].

Faced with such expectations, it seems relevant to investigate as how the extra dimensions could interfere in the energy level of the Rydberg states in the braneworld scenario. The effects of gravitational interaction on the $S$-states have already been considered before [22]. An interesting point to mention here is that when the space has more than two extra dimensions, the mean gravitational potential energy of a hydrogen-like atom in a $S$-state, $\langle U\rangle_{S}$, diverges if the brane structure is not taken into account. Indeed, this is connected to fact that the gravitational potential, $\varphi$, produced by the proton mass is not computable in the interior of the nucleus in the approximation of zero-width brane $[9,22,31]$.

On the other hand, when the atom is in a Rydberg state the situation may be different. For a state with a high enough angular momentum, the leading term of the mean gravitational potential energy, $\langle U\rangle_{R y}$, is finite in the thin brane limit. So the zero-width brane is a valid idealization here. However, under such circumstances, the amplification of the gravitational potential energy by extra dimensions is significant only if the compactification radius is greater than the atomic Bohr radius. Thus, considering the current constraints on $R$ [14], at the first sight, we could be led to think that extra dimensions would have little influence on Rydberg states.
Nevertheless, it is important to realize that the geometry around the nucleus should be similar to the ReissnerNordstrom geometry, due to the nucleus electric charge. In a Reissner-Nordstrom spacetime, besides the gravitational potential, $\varphi$, produced by the rest mass, there is also the gravitational influence associated to the energy of the electromagnetic field created by the electric charge of the source, which, in the weak field regime, can be described by a certain potential $\chi$.

Although, in the traditional three-dimensional picture, $\chi$ is smaller than the potential $\varphi$, this relation in the braneworld scenario can be different in a certain region outside the nucleus. As the electromagnetic field is spread in the threedimensional space, the potential $\chi$ diverges in every point of the brane, in the zero-thickness limit. Thus, in order to compute $\chi$, the distribution of the electric energy inside the brane should be considered. Addressing this problem in the thick brane scenario, we consider the Green function for the gravitational potential associated to length scales smaller than $R$, where the effects of extra dimensions are stronger. With this approximated Green function, we determine the short-distance potential $\chi_{s}$. We find that it can be greater than the short-distance potential $\varphi_{s}$ outside the nucleus. This can lead to interesting consequences. Indeed, considering a Hydrogen-like ion in a Rydberg state, we show that the extra dimensions can amplify the gravitational potential energy of the ion even when the compactification scale $R$ is smaller than the Bohr radius, due to the behavior of $\chi_{s}$.

Finally, we estimate the effects of higher-dimensional gravity on particular optical transitions of Hydrogen-like ions.

\section{The gravitational field produced by the nucleus}

In the original ADD-braneworld model [1], the spacetime has $\delta$ additional spacelike dimensions with the topology of a torus $T^{\delta}$. In the ground state of the model, the extra dimensions have a certain radius $R$, and it is assumed that the metric is flat. This means that the energy of the brane itself does not curve the bulk at long distances compared to the length scale of the brane, by the intervention of some mechanism [1,2]. It is also admitted that the gravitational field produced by the matter confined in the brane is governed by the EinsteinHilbert action extended to higher dimensions:

$$
S_{G}=\frac{c^{3}}{16 \pi G_{D}} \int d^{4} x d^{\delta} z \sqrt{-\hat{g}} \hat{\mathcal{R}}
$$

where $\hat{\mathcal{R}}$ is the scalar curvature of the bulk, $\hat{g}$ is the determinant of the metric whose signature is assumed to be $(-,+, \ldots,+)$ and $G_{D}$ is the gravitational constant defined in the higher-dimensional space. The coordinates $x$ and $z$ 
refer to parallel and transversal directions with respect to the brane. Due to the topology of the supplementary space, the metric is periodic in the extra directions and can be expanded in a Fourier series with respect to the $z$-coordinates, giving rise to the so-called KK-modes. The zero-mode is supposed to reproduce the four-dimensional gravitational field at large distance in comparison to $R$. This condition requires that $G_{D}$ should be related to the Newtonian gravitational constant $G$ according to the following formula $[1,9]$ :

$G_{D}=G(2 \pi R)^{\delta}$

Additionally, in order to get the correct Newtonian limit, it is also necessary that some mechanism ensures the stabilization of the volume of the supplementary space at large distance $[35,36]$.

The extremization of the action (1) yields the higherdimensional version of the Einstein equations. In the weak field regime, which we assume to be valid in the atomic domain, the metric can be written as $g_{A B}=\eta_{A B}+h_{A B}$. Here the capital Latin indices run from 0 to $3+\delta, \eta_{A B}$ is the Minkowski metric and $h_{A B}$ is a small perturbation of the order of $G_{D} M$. In a coordinate system in which the gauge condition

$\partial_{A}\left(h^{A B}-\frac{1}{2} \eta^{A B} h_{C}^{C}\right)=0$

is satisfied, the linearized equations reduce to the form

$\square h_{A B}=-\frac{16 \pi G_{D}}{c^{4}} \bar{T}_{A B}$,

where $\square$ is the D'Alembertian operator associated to the Minkowski metric and $\bar{T}_{A B}=\left[T_{A B}-(\delta+2)^{-1} \eta_{A B} T_{C}^{C}\right]$ is defined in terms of the energy-momentum tensor $T_{A B}$ of the source.

Considering the topology $\mathbb{R}^{3} \times T^{\delta}$, the solution of equation (4) for static sources is

$$
\begin{aligned}
h_{A B}(\vec{X})= & \frac{16 \pi G_{D} \Gamma\left(\frac{\delta+3}{2}\right)}{(\delta+1) 2 \pi^{(\delta+3) / 2} c^{4}} \sum_{i} \\
& \times\left(\int \frac{\bar{T}_{A B}\left(\vec{X}^{\prime}\right)}{\left|\vec{X}-\left(\vec{X}^{\prime}+\vec{K}_{i}\right)\right|^{1+\delta}} d^{3+\delta} X^{\prime}\right),
\end{aligned}
$$

where $\vec{X}=(\vec{x}, \vec{z}), \vec{K}_{i}=2 \pi R\left(0,0,0, k_{1}, \ldots, k_{\delta}\right)$ and each $k_{i}$ is an integer number. If we consider $T^{\delta}$ as a manifold embedded in $\mathbb{R}^{\delta}$, then the vectors $\vec{K}_{i}$ may be viewed as the localization of the mirror images of the source induced by the topology of the supplementary space on $\mathbb{R}^{\delta}$ (the covering space). The presence of the mirror images makes the solution periodic with respect to the $z$-coordinates as demanded by the topology. It can be shown that the higher-dimensional
Green function recovers the four-dimensional behavior for long distances $|\vec{x}|>>R$ [33]. On the other hand, in short distance $\left(\left|\vec{x}-\vec{x}^{\prime}\right|<R\right)$, the Green function is dominated by the first term of the expansion (5). In this paper, we are interested in studying the effects of the short-distance behavior of the gravitational interaction on the atomic energy levels. Thus for the sake of simplicity, we are going to take only the first term $\left(\vec{K}_{0}=0\right)$ of the series (5) as an approximation of the solution. Of course, this approximation gives a low estimate of the gravitational effect, since all terms of the series (5) we are neglecting have the same sign.

It is also important to mention here that in order to be phenomenologically consistent the theory should provide some mechanism to stabilize the volume of the supplementary space. There are different mechanisms which can ensure an appropriate behavior of the radius of the extra dimension. In general, the effect of the stabilization is to generate some mass for the radion field [35-38]. This is necessary because long-range effects $(r>>R$ ) of a massless radion field would lead to an effective gravitational coupling with a different value from the Newtonian constant $G_{N}$ [1]. For a large class of stabilization mechanisms the radion mass $m$ is smaller than the scale of the compactification $1 / R$ [35-38]. This means that gravitational effects of the radion can extend to a length scale greater than that of KK-modes of the graviton and, in principle, could be tested by Cavendish-type experiments [32,36,39]. However in the short range $r<<R$, the effects of the radion mass is negligible. Indeed, in the short-distance domain, the supplementary directions could be treated as a space with the non-compact topology $\mathbb{R}^{\delta}$ and, therefore, the green function for gravitational field reduces to the first term of (5). This is the usual procedure adopted in the study of black-hole production in colliders [40-42]. In this paper, as we are studying the effects of the short-distance gravitational potential, then the mass of the radion can be ignored in the following calculations.

In the context of the braneworld, it is assumed that the energy-momentum tensor of the confined fields has the form [9]:

$T_{A B}(x, z)=\eta_{A}^{\mu} \eta_{B}^{\nu} T_{\mu \nu}(x) f(z)$,

where $T_{\mu \nu}(x)$ is the ordinary energy-momentum tensor of the four-dimensional fields that live in the brane and $f(z)$ is a normalized distribution very concentrated around the brane, which is approximately given by a delta-like distribution in the thin brane limit. The function $f(z)$ describes the confinement of a field in the brane and, in principle, it could be a different function for each type of field.

In our system, the atomic nucleus is the source of the gravitational field. Due to its electric charge, it is reasonable to expect that the spacetime geometry around the nucleus should be similar to the Reissner-Nordstrom geometry. Based 
on this, it is convenient to decompose the energy-momentum tensor as a sum of two terms: $T_{\mu \nu}=T_{\mu \nu}^{(0)}+T_{\mu \nu}^{(E M)}$, where $T_{\mu \nu}^{(0)}$ describes the rest energy concentrated inside the nucleus and $T_{\mu \nu}^{(E M)}$ represents the stress-energy tensor of the electromagnetic field created by the charge, which is spread in the space.

We deal with each term separately. The first tensor can be written as $T_{A B}^{(0)}=c^{2} \rho \eta_{A}^{0} \eta_{B}^{0}$, where $\rho$ is the mass density of the source. On its turn, in SI units, $T_{\mu \nu}^{(E M)}=$ $\epsilon_{0} c^{2}\left(F_{\mu \lambda} F_{\nu}{ }^{\lambda}-\frac{1}{4} \eta_{\mu \nu} F_{\alpha \beta} F^{\alpha \beta}\right)$, where $F_{\mu \nu}$ is the electromagnetic tensor and $\epsilon_{0}$ is the electric permittivity of the free space. In a first approach, let us ignore the contribution of the magnetic field produced by the proton's magnetic dipole. In this approximation, only the components of the electric field, $F_{0 i}=E_{i} / c$, are non-null (in the zero order of $G_{D}$ ). Take into account all this consideration, we can show that the metric can be written as:

$$
\begin{aligned}
d s^{2}= & -\left(1+\frac{2}{c^{2}} \varphi_{s}+\frac{2(2+\delta)}{c^{2}(1+\delta)} \chi_{s}\right)\left(d x^{0}\right)^{2} \\
& +\left(1-\frac{2}{c^{2}(1+\delta)} \varphi_{s}\right)\left[\left(1+\frac{2(2+\delta)}{c^{2}(1+\delta)} \lambda_{1, s}\right) d r^{2}\right. \\
& \left.+\left(1+\frac{2(2+\delta)}{c^{2}(1+\delta)} \lambda_{2, s}\right) r^{2}\left(d \theta^{2}+\sin ^{2} \theta d \phi^{2}\right)\right] \\
& +\left(1-\frac{2}{c^{2}(1+\delta)} \varphi_{s}\right) d \vec{z}^{2},
\end{aligned}
$$

where $x^{0}=c t$, the coordinates $(r, \theta, \phi)$ are the usual spherical coordinates associated to the "almost Cartesian" coordinates $\left(x^{1}, x^{2}, x^{3}\right)$, defined by Eq. (3). The function $\varphi_{s}$ plays the role of a Newtonian potential produced by the nuclear mass in $\mathbb{R}^{3+\delta}$ :

$\varphi_{s}(\vec{X})=-\hat{G}_{D} \int \frac{\rho\left(\vec{x}^{\prime}\right) f_{m}(z)}{\left|\vec{X}-\vec{X}^{\prime}\right|^{1+\delta}} d^{3+\delta} X^{\prime}$,

where $\hat{G}_{D}=4 G_{D} \Gamma\left(\frac{3+\delta}{2}\right) /\left[(2+\delta) \pi^{(1+\delta) / 2}\right]$. The sub-index $s$ emphasizes that $\varphi_{s}$ is the potential given by the shortdistance Green function, which corresponds to first term of the series (5). Analogously, $\chi_{s}$ is the short-distance gravitational potential produced by the energy of the electromagnetic field $u=\left(\epsilon_{0} E^{2} / 2\right)$ created by the electric charge:

$\chi_{s}(\vec{X})=-\frac{\hat{G}_{D}}{c^{2}} \int \frac{u\left(\vec{x}^{\prime}\right) f_{e}(z)}{\left|\vec{X}-\vec{X}^{\prime}\right|^{1+\delta}} d^{3+\delta} X^{\prime}$

The spatial components, $T_{i j}^{(E M)}$, of the electromagnetic stress-energy tensor, give rise to the functions $\lambda_{2, s}$ and $\lambda_{1, s}$, which are defined by:

$$
\begin{aligned}
\lambda_{2, s}= & -\chi_{s}-\pi \frac{\hat{G}_{D}}{c^{2}} \int \frac{\epsilon_{0} E^{2} r^{\prime 2}\left(\sin ^{3} \theta\right) d r^{\prime} d \theta}{\left.\left.\left|\left(r^{2}+r^{\prime 2}-2 r r^{\prime} \cos \theta\right)+\right| \vec{z}\right|^{2}\right|^{\frac{1+\delta}{2}}} \\
& \times f_{e}(z) d^{\delta} z^{\prime}, \\
\lambda_{1, s}= & -\chi_{s}-2 \pi \frac{\hat{G}_{D}}{c^{2}} \int \frac{\epsilon_{0} E^{2} r^{\prime 2}\left(\cos ^{2} \theta \sin \theta\right) d r^{\prime} d \theta}{\left.\left.\left|\left(r^{2}+r^{\prime 2}-2 r r^{\prime} \cos \theta\right)+\right| \vec{z}\right|^{2}\right|^{\frac{1+\delta}{2}}} \\
& \times f_{e}(z) d^{\delta} z^{\prime} .
\end{aligned}
$$

As we shall see in the next section, in the gravitational sector of the atomic Hamiltonian $\left(H_{G}\right)$, the leading term comes from the component $g_{00}$, which depends on the potential $\varphi_{s}$ and $\chi_{s}$. To determine explicitly the functions $\varphi_{s}$ and $\chi_{s}$, it is important to look at the internal structure of the brane to see how the fields are localized inside. In a field-theoretic framework, topological defects are possible realizations of a brane, since these structures are capable of localizing fermions in their cores, as illustrated in Ref. [34], where it is shown that Dirac fields can be trapped inside a domain wall by means of a Yukawa-like interaction. In this context, usually known as thick brane scenario, a delta-like localization is replaced by a non-singular confinement where the fermion's states are described by a regular wave-function with a tiny width $\sigma$ in the transversal directions.

Following these ideas, in Ref. [22], we calculated the gravitational potential, $\varphi_{s}$, produced by the proton mass $M_{p}$ in the thick brane scenario. By admitting that the proton wavefunction in the transversal directions has a Gaussian profile, we have estimated the influence of the gravitational interaction on the energy of $S$-states of a Hydrogen atom. The leading contribution is proportional to $\hat{G}_{D} m M_{p} / a_{0}^{3} \sigma^{\delta-2}$, where $m$ is the electron mass and $a_{0}$ is the Bohr radius. It is clear, from this expression, that, the calculation diverges, even at the tree level, in the thin brane limit. Therefore, the mass distribution of the nucleus inside the brane cannot be neglected when the atom is in $S$-states. We should highlight that the mentioned term corresponds to the mean value of the gravitational potential energy integrated in the interior of the nucleus, which we shall denote as $\left\langle H_{G}\right\rangle_{i n}$. On its turn, outside the nucleus, the dominant contribution is proportional to $\hat{G}_{D} M m / a_{0}^{1+\delta}$, if the compactification scale is greater than the Bohr radius. As, in realistic scenarios, $\sigma<<a_{0}$ then the interior contribution $\left\langle H_{G}\right\rangle_{i n}$ is much greater than the exterior contribution $\left\langle H_{G}\right\rangle_{\text {out }}$ for the energy of $S$-states.

However, if the atom is in a Rydberg states with large angular momentum, the inverse happens. Indeed, in states with angular momentum $l$, the internal contribution is reduced by a factor of the order of $\left(r_{N} / a_{0}\right)^{2 l}$, where $r_{N}$ is the radius of the nucleus. Therefore, for some angular momentum higher than 
$2 l>(\delta-2) \frac{\ln \left(a_{0} / \sigma\right)}{\ln \left(a_{0} / r_{N}\right)}$,

the exterior contribution $\left\langle H_{G}\right\rangle_{\text {out }}$ become greater than $\left\langle H_{G}\right\rangle_{\text {in }}$. Therefore, as the leading term does not depend on the brane thickness, we may say that a zero-width brane is a valid approximation for such states with high quantum numbers. Replacing $f_{m}(z)$ in Eq. (8) by a delta-Dirac distribution, we find that, in the exterior region, the short-distance gravitational potential produced by the nucleus mass is

$\varphi_{s}=-\hat{G}_{D} \frac{M}{r^{1+\delta}}$.

With respect to the complete potential given by the series (5), the term (13) is the leading contribution in the region $r_{N}<<$ $r<<R$. By using (2), we can conclude that the contribution of (13) to the gravitational potential energy of the atom in a Rydberg state will be of the order of $\left(G M m / a_{0}\right)\left(R / a_{0}\right)^{\delta}$ and, therefore, extra dimensions would amplify significantly the gravitational energy of the atom in a Rydberg state only if $R>>a_{0}$.

This conclusion is not necessarily valid when we consider the potential $\chi_{s}$ produced by the electromagnetic energy. If $f_{e}(z)$ is approximated by a delta-like distribution in Eq. (9), the potential $\chi_{s}$ diverges everywhere, not only inside the nucleus, as happens with the potential $\varphi_{s}$, since the electromagnetic field is spread in the space. Therefore, due to the behavior of potential $\chi_{s}$, the zero-width brane is not a valid idealization even when we are dealing with ions in Rydberg states.

Thus, in order to compute $\chi_{s}$, we have to consider the distribution of the electric energy inside the thick brane. With the purpose of obtaining some estimates, we are going to admit that the electric energy is uniformly distributed inside a compact region of the brane with a size $\varepsilon$, which may have the same order of the brane thickness. Thus, if $V_{\delta}(\varepsilon)$ denotes the volume of a ball with a radius $\varepsilon$ in the supplementary space, $f_{e}(z)$ can be defined as the step function:

$f_{e}(z)= \begin{cases}1 / V_{\delta}(\varepsilon), & z \leq \varepsilon, \\ 0, & z>\varepsilon .\end{cases}$

Taking this function in the expression (9) and integrating it with respect to the angular coordinates, the potential $\chi_{s}$ evaluated in the brane $(\vec{z}=0)$ can be written as $\chi_{s}=\chi_{+}-$ $\chi_{-}$, where

$$
\begin{aligned}
\chi_{ \pm}(r)= & \frac{\hat{G}_{D} S_{\delta}}{V_{\delta}(\varepsilon)} \frac{2 \pi}{(\delta-1)} \frac{1}{r} \int \frac{u\left(r^{\prime}\right)}{\left[\left(r \pm r^{\prime}\right)^{2}+z^{\prime 2}\right]^{\frac{-1+\delta}{2}}}\left(z^{\prime}\right)^{\delta-1} \\
& \times r^{\prime} d r^{\prime} d z^{\prime}
\end{aligned}
$$

Here $S_{\delta}$ is the hyper-area of a spherical hypersurface of $\delta$ dimensions with a unit radius. In (15), the integration interval of the transversal variable is $0<z^{\prime}<\varepsilon$.

The divergent term in the thin brane limit comes from the function $\chi_{-}$as the integrating variable $r^{\prime}$ passes through $r$. In the context of a thick brane scenario, this "dangerous" term can be isolated and calculated explicitly. As we are interested in studying Rydberg states with high angular momentum, we may restrict our analysis to points far from the nucleus $(r>>$ $\left.r_{N}\right)$. In the calculation of $\chi_{s}$, it is convenient to separate the integration domain in two parts: the region inside the interval $\left|r^{\prime}-r\right| \leq R$, where the short-distance behavior of the Green function is dominant, and the external region $\Omega$. Noticing that the zero-thick brane approximation is valid in $\Omega$, we can write:

$$
\begin{aligned}
\chi_{s}(r) \approx & \frac{2 \pi \hat{G}_{D}}{(\delta-1)} \frac{1}{r} \int_{\Omega}\left[\frac{1}{\left|r+r^{\prime}\right|^{-1+\delta}}-\frac{1}{\left|r-r^{\prime}\right|^{-1+\delta}}\right] \\
& \times u\left(r^{\prime}\right) r^{\prime} d r^{\prime} \\
& -\frac{\hat{G}_{D} S_{\delta}}{V_{\delta}(\varepsilon)} \frac{2 \pi}{(\delta-1)} \frac{1}{r} \int_{\left|r^{\prime}-r\right| \leq R} \frac{u\left(r^{\prime}\right)}{\left[\left(r \pm r^{\prime}\right)^{2}+z^{\prime 2}\right]^{\frac{-1+\delta}{2}}} \\
& \times\left(z^{\prime}\right)^{\delta-1} r^{\prime} d r^{\prime} d z^{\prime} .
\end{aligned}
$$

To proceed further we need now to specify the electromagnetic energy distribution in the three-dimensional space by means of the function $u$. To be consistent, the model should reproduce the ordinary behavior of electromagnetic field in a length scale greater than the brane thickness $(r>>\varepsilon)$. It is important to remark at this point that the compactification scale $(R)$ is admitted to be greater than $\varepsilon$ in our approach, so the gravitational field is the only field directly affected by extra dimensions in the region $r>>\varepsilon$. Based on this considerations, we will assume that in the three-dimensional space the energy density of the electric field has the usual form given by:

$u=\frac{1}{2} \epsilon_{0} E^{2}=\frac{Q^{2}}{32 \pi^{2} \epsilon_{0}} \times\left\{\begin{array}{l}\frac{r^{\prime 2}}{r_{N}^{6}}, r^{\prime} \leq r_{N} \\ \frac{1}{r^{\prime 4}}, r^{\prime} \geq r_{N}\end{array}\right.$

which is the well-known energy of electromagnetic field produced by a charge $Q$ uniformly distributed inside a ball of radius $r_{N}$ in three-dimensional space. With this energy distribution, we find:

$\chi_{s}=-\frac{\beta_{\delta}}{16 \pi \epsilon_{0} c^{2}} \frac{\hat{G}_{D} Q^{2}}{\varepsilon^{\delta-2} r^{4}}+\mathcal{O}_{1}+\mathcal{O}_{2}$,

where $\beta_{\delta}$ can be written in terms of the gamma function as:

$\beta_{\delta}=\frac{\delta}{(\delta-1)} \frac{\sqrt{\pi} \Gamma\left(\frac{\delta-2}{2}\right)}{2 \Gamma\left(\frac{\delta-1}{2}\right)}$, 
and the corrections terms are of the following order:

$$
\begin{aligned}
& \mathcal{O}_{1} \sim O\left(\frac{\varepsilon}{R}\right)^{\delta-2} \times\left\{\begin{array}{l}
1+O(R / r)^{2}, \text { if } \delta \text { is odd } \\
O(R / r), \text { if } \delta \text { is even }
\end{array} ;\right. \\
& \mathcal{O}_{2} \sim O\left(\frac{\varepsilon}{r_{N}}\right)^{\delta-2} \times O\left(r_{N} / r\right)^{\delta-1} .
\end{aligned}
$$

At this point, it is important to remark that the exact profile of the electromagnetic energy distribution inside the brane, given by the function $f_{e}(z)$, should depend on the localization mechanism of the gauge field. However, it is interesting to mention that for any normalized function $f_{e}(z)$, the potential $\chi_{s}$, in the leading order, has exactly the same form given by (18), provided that the parameter $\varepsilon$ be defined as

$\frac{1}{\varepsilon^{\delta-2}}=\frac{2}{\delta} \int \frac{f_{e}(\vec{z})}{|\vec{z}|^{\delta-2}} d^{\delta} z$.

Therefore, from the phenomenological point of view, the gravitational potential $\chi_{s}$, in leading order, does not depend on many details of the localized fields, but only on a specific moment $\left\langle 1 / z^{\delta-2}\right\rangle_{f}$ of the distribution $f_{e}$, i.e., on the average value of $1 / z^{\delta-2}$ with respect to the distribution $f_{e}(\vec{z})$. As $f_{e}(z)$ should be concentrated inside the brane, it is reasonable to expect that $\varepsilon$ is related to the brane thickness.

Now, we can compare the strength of the potentials $\chi_{s}$ and $\varphi_{s}$. We can see that $\chi_{s}$ is greater than $\varphi_{s}$ if

$r>\left[\frac{4}{\beta_{\delta}} \frac{M c^{2}}{Q^{2} / 4 \pi \epsilon_{0} a_{0}}\left(\frac{\varepsilon}{a_{0}}\right)\right]^{\frac{1}{\delta-3}} \varepsilon$.

For realistic values of $\varepsilon$, the above condition is satisfied outside the nucleus. So, in this scenario, the gravitational potential produced by the electromagnetic field is greater than the short-distance term of the gravitational potential of the nuclear mass, in the exterior region.

In three-dimensional space, this does not happen. Indeed, considering the same distribution (17), we find in the exterior region: $\chi_{(3)}=-G E /\left(c^{2} r\right)+G Q^{2} /\left(4 \pi \epsilon_{0} c^{2} r^{2}\right)$, where $E$ is proportional to the energy of the electromagnetic field. The attractive term of $\chi_{(3)}$ depends on the inverse of the distance $r$, therefore, it can be incorporated in the three-dimensional gravitational potential $\varphi_{(3)}$ by absorbing the electromagnetic energy as part of the rest energy of the system. It follows then that the potential, $\varphi_{(3)}=-G M / r$, with $M=M_{0}+E / c^{2}$, is greater than the repulsive part of the potential $\chi_{(3)}$ outside the nucleus.

At this point, let us mention that the mass distribution of the nucleus could be described by a continuous density $\rho_{m}$, instead of a compact distribution, without changing our conclusion. In this new configuration, the potential $\varphi_{s}$ would diverge everywhere in the thin brane limit, but, in the thick brane scenario, the "dangerous" term would be proportional to $\hat{G}_{D} \rho_{m} / \sigma^{\delta-2}$. Thus, it will be smaller than $\chi_{s}$ for $r>>$ $r_{N}$, provided that $\rho_{m}$ is a fast decreasing function, like an exponential.

In this section, we have calculated the gravitational field produced by the nucleus. In order to obtain finite results, it was necessary to take into account the distribution of the nuclear mass and the electromagnetic field inside the brane. In the next sections, we intend to discuss the complementary aspect of the picture, namely, the influence of gravity on the dynamics of the electromagnetic and the Dirac fields in this scenario.

\section{The electrostatic potential on the brane}

As we have mentioned before, in the ADD-model, it is assumed that the effects of extra dimensions appears first in the gravitational field, since it can spread into the extraspace, which has a size $R$ that is much greater the brane width $\varepsilon$, where the other fields are confined. So it is reasonable to expect that direct effects of extra dimension on the other fields would be smaller than the gravitational effect by a factor of the order of $(\varepsilon / R)^{\delta}$. Thus, in a first approximation picture, we may assume, as usual, that the standard model fields can be treated as traditional four-dimensional fields confined in a 3-brane.

Based on this considerations, it follows that, in length scales above the brane thickness, the fields do not couple to the bulk geometry directly, but their dynamic in the spacetime is influenced by gravity through the brane geometry. According to the present approximation scheme, the geometry of the 3-brane is isometric to the geometry of thick brane center (i.e., the submanifold $z=0$ ), since any deviations in the metric components inside the brane are negligible given that they are of the order of $\varepsilon$, at least. Thus, in the given coordinate system, the induced metric on the submanifold $z=0$ can be directly obtained from (7). After a transformation to isotropic coordinates, the induced metric can be written in the following form:

$d s^{2}=-w^{2}\left(d x^{0}\right)^{2}+v^{2}(d \vec{x} \cdot d \vec{x})$,

where

$$
\begin{aligned}
w^{2}=1 & +\frac{2}{c^{2}} \varphi_{s}+\frac{2(2+\delta)}{c^{2}(1+\delta)} \chi_{s}, \\
v^{2}= & 1-\frac{2}{c^{2}(1+\delta)} \varphi_{s}+\frac{2(2+\delta)}{c^{2}(1+\delta)} \\
& \times\left[\lambda_{2, s}+\int \frac{\lambda_{2, s}-\lambda_{1, s}}{r} d r\right] .
\end{aligned}
$$


In this context, let us determine the modification on the electrostatic interaction between the electron and nucleus caused by the geometry of the brane. The Maxwell equation in the curved space can be written as

$$
\frac{1}{\sqrt{-g}} \frac{\partial}{\partial x^{\mu}}\left(\sqrt{-g} F^{\mu \nu}\right)=\mu_{0} J^{v},
$$

where $g$ is the determinant of the metric tensor (22), $J^{v}$ is the four-current and $\mu_{0}$ is the vacuum magnetic permeability. If $U^{\mu}=d x^{\mu} / d \tau$ is the four-velocity field of the source, then $J^{\mu}=-\rho_{e} U^{\mu}$, where $\rho_{e}$ is the proper charge density and $\tau$ is the proper time. For the sake of simplicity, let us admit that the nucleus is at rest in the given frame. Thus, the normalized four-velocity has the following components: $U^{\mu}=\left(c w^{-1}, 0\right)$, in the given coordinate system.

In the static regime, the four-potential reduces to $A_{\mu}=$ $(-\phi / c, 0)$. Considering that $F_{\mu \nu}=\partial_{\mu} A_{\nu}-\partial_{\nu} A_{\mu}$, we can derive from (25) the field equation for the electric potential $\phi$ :

$\partial_{i}\left(\frac{v}{w} \partial^{i} \phi\right)=-\frac{\rho_{e, 0}}{\epsilon_{0}}$

where $\rho_{e, 0}=v^{3} \rho_{e}$ is the charge density with the flat measure $d^{3} x$. This means that in a hypersurface orthogonal to $U^{\mu}$, it satisfies the condition: $\rho_{e, 0}\left(d^{3} x\right)=\rho_{e}\left(v^{3} d^{3} x\right)$.

As the gravitational field is weak, we expect that the gravitationally modified electric potential can be written as $\phi=\phi_{0}+\phi_{G}$, where $\phi_{0}$ is the solution in the flat space sourced by $\rho_{e, 0}$ and $\phi_{G}$ is a small correction of the order of $G_{D}$ due to the spacetime curvature. In the first order of $G_{D}$, the field Eq. (26) reduces to the form

${ }^{0} \nabla^{2} \phi_{G}=-\frac{\rho_{P}}{\epsilon_{0}}$

where ${ }^{0} \nabla^{2}$ is the Laplacian in the flat three-dimensional space and

$\rho_{P}=\nabla \cdot\left[(1-v / w) \epsilon_{0} \vec{E}\right]$

plays the role of a polarization charge density which works here as a source of the gravitational correction of the electrostatic potential. The solution is

$\phi_{G}(x)=\frac{1}{4 \pi \epsilon_{0}} \int \frac{\rho_{P}\left(x^{\prime}\right)}{\left|\vec{x}-\vec{x}^{\prime}\right|} d^{3} x^{\prime}$

which is proportional to $\hat{G}_{D} M Q^{2} / c^{4}$ in the leading order.

\section{Dirac equation on the brane}

The localization of spinors fields in the core of topological defects is a very interesting and involving problem [34,4345]. In Ref. [34], this localization mechanism is illustrated by a procedure that is capable of trapping a five-dimensional massless spinor in the center of a domain wall. Due to a Yukawa-type interaction between the spinor and scalar field that describes the wall, there is a chiral zero-mode Weyl spinor which is normalizable and localized in the center of brane. It is assumed that this localized spinor, which acquires some mass by means of another mechanism, describes the matter in the low-energy regime.

The fundamental theory in high-energy regime could be very complex and possibly contains additional degrees of freedom (the KK-modes, for instance). However, whatever the theory in the high-energy regime, the localization mechanism must ensure that the long-range degrees of freedom should reproduce the known four-dimensional behavior $[31,46]$.

Based on this phenomenological perspective, we are going to assume here that at a length scale greater than the brane thickness, the matter is described by four-dimensional Dirac fields confined to the center of the brane. Thus, to take into account the gravitational interaction between fermions, we shall admit that the confined particles do not feel the geometry of the bulk, but instead they interact with the metric of the $(3+1)$-spacetime given in $(22)$.

Associated with that metric $g_{\mu \nu}$, we find a set of four orthonormal vector fields, whose components, $e_{\hat{a}}^{\mu}(x)$ (the vierbein fields), satisfy the equations $g_{\mu \nu} e_{\hat{a}}^{\mu} e_{\hat{b}}^{\nu}=\eta_{\hat{a} \hat{b}}$. Here the indices $\hat{a}$ and $\hat{b}$ identifies the vector fields and $\mu$ and $v$ refer to their components with respect to the given coordinate system. The indices run from 0 to 3 . From the four Dirac matrices $\left(\gamma^{a}\right)$ defined in the Minkowski space and with the help of the vierbein fields, we can construct the matrices $\gamma^{\mu}(x)=e_{\hat{a}}^{\mu}(x) \gamma^{a}$ which satisfy the anti-commutation relation $\left\{\gamma^{\mu}(x), \gamma^{v}(x)\right\}=2 g^{\mu \nu}(x)$ in the curved space.

Assuming a minimal coupling with the gravitational field, the Dirac equation (in a curved space) that describes the state of a fermion with mass $m$ and electric charge $q$ subject to gauge field $A_{\mu}$ is given by:

$\left[i \gamma^{\mu}(x) D_{\mu}-m c / \hbar\right] \psi(x)=0$,

where the operator $D_{\mu}=\nabla_{\mu}-i q A_{\mu}$ and $\nabla_{\mu}$ is the covariant derivative of the spinor, which can be written in terms of the spinorial connection $\Gamma_{\mu}(x)$ as

$\nabla_{\mu} \psi(x)=\left[\partial_{\mu}+\Gamma_{\mu}(x)\right] \psi(x)$. 
On its turn, $\Gamma_{\mu}(x)$ is defined as

$\Gamma_{\mu}(x)=-\frac{i}{4} \sigma^{a b} e_{\hat{a}}^{v}(x) e_{\hat{b} v ; \mu}(x)$,

where $\sigma^{a b}=\frac{i}{2}\left[\gamma^{a}, \gamma^{b}\right]$ is a representation of the Lorentz Lie Algebra in the spinor space. As usual, the symbol [,] is the commutator operator and $e_{b v ; \mu}(x)$ is the covariant derivative of the vierbein fields which depend on the LeviCivita connection $\Gamma_{v \mu}^{\lambda}$ (the affine connection compatible with the metric) according to

$e_{\hat{b} v ; \mu}(x)=\partial_{\mu} e_{\hat{b} v}(x)-\Gamma_{v \mu}^{\lambda}(x) e_{\hat{b} \lambda}(x)$.

For the diagonal metric (22), a possible choice for the vierbein fields are:

$e_{\hat{0}}^{0}(x)=w^{-1}$,

$e_{\hat{\jmath}}^{i}(x)=\delta_{j}^{i} v^{-1}$,

and all the other components equal to zero.

By a direct calculation, the Dirac equation can be written in the form $i \hbar \frac{\partial \psi}{\partial t}=H \psi$. The operator $H$ is the atomic Hamiltonian, which, in a convenient representation, assumes the following form in the first order of $G_{D}$ [47]:

$H=\frac{1}{2}\left\{\vec{\alpha} \cdot \vec{p}, \frac{w}{v}\right\}+w \beta m c^{2}+q \phi$.

Here $\vec{p}$ is the usual three-dimensional momentum operator in flat spacetime, $\alpha^{i}=\gamma^{0} \gamma^{i}$ and $\beta=\gamma^{0}$. For the sake of simplicity, we are neglecting the effects of the potential vector $\vec{A}$. It is also important to mention that, in the chosen representation, the Hamiltonian (36) is Hermitian in the Hilbert space of square-integrable functions endowed with the usual inner product calculated with the flat measure $d^{3} x$.

Following the Foldy-Wouthuysen procedure, the nonrelativistic limit of the Hamiltonian (36) can be obtained, by admitting that the mean value of each term of $H$ is much smaller than the rest energy of the test particle, $m c^{2}$, in Rydberg states. In the gravitational sector of the Hamiltonian, i.e., in the part of $H$ constituted by terms proportional to $G_{D}$, we find that the leading terms are proportional to the test particle mass. Thus, in this approximation, we can write:

$H_{G} \approx m \varphi_{s}+\frac{2(2+\delta)}{(1+\delta)} m \chi_{s}$

As $\chi_{s}$ is greater than $\varphi_{s}$ in the exterior region, then we can consider a further approximation:

$H_{G} \approx \frac{2(2+\delta)}{(1+\delta)} m \chi_{s}$, in order to find corrections on the energy levels of Rydberg states, coming from the short-distance behavior of the higherdimensional gravitational field produced by the energy of the electrovacuum surrounding the test particle in a Hydrogenlike ion.

\section{Results and discussion}

Treating $H_{G}$ as a small term of the total Hamiltonian, we can use the perturbation method to estimate the gravitational potential energy of a Hydrogen-like ion in a Rydberg state. For an ion with an atomic number $Z$ found in a state whose principal number is $n$ and $l$ is the angular momentum, the mean gravitational potential energy is approximately given by

$\left\langle n, l\left|H_{G}\right| n, l\right\rangle=-\hat{\beta}_{\delta} \gamma_{n, l} \frac{G_{D} Z^{4} e^{2} m}{c^{2} \varepsilon^{\delta-2} a_{0}^{4} \epsilon_{0}}$,

where $e$ is the fundamental electric charge and

$$
\begin{aligned}
\hat{\beta}_{\delta} & =\frac{\delta \Gamma\left(\frac{\delta-2}{2}\right)}{4 \pi^{(2+\delta) / 2}}, \\
\gamma_{n, l} & =\frac{\left(3 n^{2}-l(l+1)\right)}{n^{5} l(l+1)(2 l+3)(2 l+1)(2 l-1)} .
\end{aligned}
$$

Actually, it is important to stress, the energy (39) is provided by the short-distance behavior of the Green function for the gravitational potential.

Without extra-dimensions, the gravitational potential energy of the ion in the same state is

$\left\langle n, l\left|H_{G}^{(3)}\right| n, l\right\rangle=-\frac{A Z}{n^{2}} \frac{G m M_{p}}{a_{0}}$,

where $A$ is the mass number. Here for the sake of simplicity, we admit that the mass of the nucleus is approximately equal to $A M_{p}$.

Comparing (39) and (41), we can find conditions under which the amount of gravitational potential energy coming from the short-distance behavior of the higher-dimensional potential surpasses the three-dimensional value. In this domain, extra dimensions may amplify significantly the gravitational effects on the ion. The condition $\left\langle H_{G}\right\rangle>\left\langle H_{G}^{(3)}\right\rangle$ can be expressed in terms of the size of the extra dimensions $R$ in the following form:

$(2 \pi R)^{\delta}>\frac{1}{\hat{\beta}_{\delta} \gamma_{n, l}} \frac{A}{Z^{3} n^{2}} \frac{\epsilon_{0} M_{p} c^{2} a_{0}^{3} \varepsilon^{\delta-2}}{e^{2}}$

Notice that, in order to the gravitational potential energy be amplified by the extra-dimension, $R$ should be some orders 
of magnitude greater than the brane thickness, but interestingly the compactification scale can be smaller than the Bohr radius. As an example, consider the Neon ion $\left({ }^{20} \mathrm{Ne}^{+9}\right)$ in a six-dimensional space. In a state with $n=15$ and $l=14$, the extra dimensions would provide an amplification if $R>10^{-14} \mathrm{~m}$, when $\varepsilon=10^{-20} \mathrm{~m}$, for instance.

Now let us consider the transition between the states $(n, l=n-1)$ and $(n-1, l=n-2)$. The principal part of the energy gap between these levels is

$\Delta E_{P}=\frac{Z^{2} e^{2}}{8 \pi \epsilon_{0} a_{0}}\left(\frac{1}{n^{2}}-\frac{1}{(n-1)^{2}}\right)$.

From (39), we can calculate the difference of the gravitational potential energy between the mentioned levels $\left(\Delta E_{G}\right)$. Relative to the principal part of the energy gap, we find

$$
\frac{\Delta E_{G}}{\Delta E_{P}}=-8 \pi \hat{\beta}_{\delta} \frac{\left(\gamma_{n, n-1}-\gamma_{n-1, n-2}\right)}{n^{-2}-(n-1)^{-2}} \frac{Z^{2} G_{D} m}{c^{2} \varepsilon^{\delta-2} a_{0}^{3}} .
$$

If we consider muonic hydrogen-like ions, then the Bohr radius of the ground-state of the Hydrogen, $a_{0}$, is replaced by the Bohr radius of the muonic Hydrogen, $a_{0, \mu}$, and the electron mass $m$ is substituted by the muon mass $m_{\mu}$. Due to this, the magnitude of expression (44) is multiplied by a factor $\left(a_{0} / a_{0, \mu}\right)^{3}\left(m_{\mu} / m\right) \sim 10^{9}$.

According to Ref. [23], if the transition frequency lies in the optical band, the relative experimental precision could reach the order of $10^{-19}$ [26]. Given this fantastic precision, it is interesting to investigate the effects of the hidden dimensions on the transitions between Rydberg states.

In a previous paper [48], it was suggested that the proton radius puzzle can be explained in the braneworld scenario. Briefly speaking, the puzzle is a discrepancy between the proton charge radius recommended by CODATA $\left(r_{p}^{(C D)}\right)-$ which is obtained from data of hydrogen and deuteron spectroscopy combined with data of electron-proton elastic scattering - and the radius inferred from the measurement of the Lamb shift of the muonic Hydrogen [27-30]. The point is that the experimental value of the energy difference between $2 S$ and $2 P$ states, in the muonic hydrogen, is approximately $0.3 \mathrm{meV}$ greater than theoretical prediction calculated with $r_{p}^{C D}$. This excess of energy, which has no explanation within the standard model of physics, may be an indication of a missing interaction between the proton and muon.

In Ref. [48], it was argued that the muon-proton gravitational interaction, modified by extra-dimensions, can account for the unexpected energy excess measured in the muonic Hydrogen Lamb shift. Considering a thick brane scenario, as described here, the mean gravitational potential of the muonic hydrogen in states $2 S$ and $2 P$ can be calculated. Then, to solve the proton radius puzzle, it is required that the difference of the gravitational potential energy be equal to

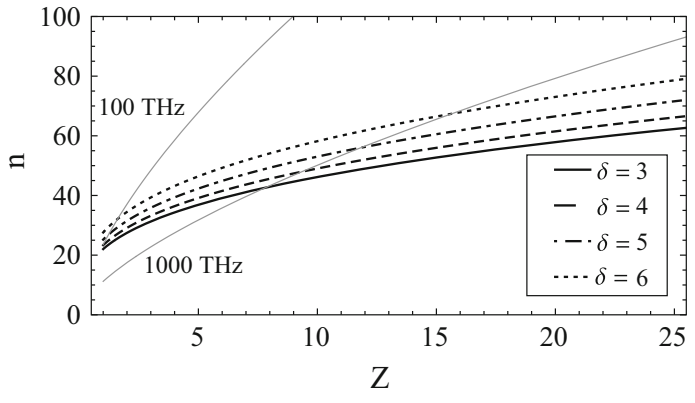

Fig. 1 In transitions between Rydberg states of muonic Hydrogenlike ions below the dashed lines, the influence of hidden dimensions, through the classical higher-dimensional gravity, can be greater than promised experimental uncertainty in measurements of transition frequencies around the optical band (100-1000 THz). Here we are assuming that $G_{D} / \sigma^{\delta-2}$ has the appropriate value to solve the proton radius puzzle [48]

energy excess of $0.3 \mathrm{meV}$. This leads to the condition:

$\frac{\gamma_{\delta}}{8 \pi} \frac{\hat{G}_{D} m_{p} m_{\mu}}{\sigma^{\delta-2} a_{0, \mu}^{3}}\left(1-\frac{3 r_{p}}{2 a_{0, \mu}}\right)=0.3290(469) \mathrm{meV}$,

where $\gamma_{\delta}$ is a factor whose values depend on the number of dimensions [48].

The Eq. (45) imposes a condition for the ratio, $\hat{G}_{D} / \sigma^{\delta-2}$, between the fundamental gravitational constant $G_{D}$ (or equivalently the fundamental Planck mass $M_{D}$ ) and the parameter $\sigma$, which here can be seen as the confinement parameter of the nucleus in the brane.

Taking this value of $\hat{G}_{D} / \sigma^{\delta-2}$ as reference in Eq. (44) and admitting that the matter and the electromagnetic fields have the same confinement parameter $(\varepsilon \simeq \sigma)$, we can make some estimates. We show in Fig. 1 what are the optical transitions between Rydberg states in which the effect of the higher-dimensional gravity exceeds the experimental precision promised by the optical metrology [26].

It is important to remark that these predictions are based on the classical behavior of the gravitational field. On the other hand, as pointed out in Ref. [9], quantum-gravity effects would become relevant at a length scale around the fundamental Planck length $\left(\ell_{D}\right)$ defined in the higher dimensions or even in greater distances. If this is the case, then quantum-gravity effects could modify the classical result unpredictably. Nevertheless, as a definite quantum-gravity theory is not yet known, only experiments can answer this question.

\section{Final remarks}

The prospect of measuring optical transitions between Rydberg states with a relative uncertainty near to the impressive 
order of $10^{-19}$ motivated us to investigate the influence of hidden dimensions on the energy levels of the Rydberg states of hydrogen-like ions.

In the braneworld scenario with large extra dimensions, gravity is the first interaction to be affected by the supplementary space. These models predict that, in comparison to the three-dimensional Newtonian interaction, the gravitational field can be hugely amplified in short distance. In this paper, we have investigated the effects of extra dimensions in the atomic domain by studying the influence of the higherdimensional gravitational field produced by the nucleus on the energy levels of the ion.

Due to the electric charge of the source, the spacetime around the nucleus is a brane-version of the ReissnerNordstrom geometry. In this kind of spacetime, the metric, in the weak field regime, depends on the gravitational potential produced by the rest energy concentrated on the nucleus, $\varphi$, and also on the gravitational potential generated by the energy of the electric field created by the nucleus charge, $\chi$.

In the zero-thickness approximation, the short-distance potential $\chi_{s}$ diverges everywhere in the brane. Due to this, the potential gravitational energy is not computable in the thin brane limit even when the ion is in Rydberg states with high angular momentum.

Therefore, in order to estimate the influence of the potential $\chi_{s}$ on the energy of the ion, we had to address this problem in the thick brane scenario. Considering a regular distribution of the electromagnetic energy inside a brane strip, we calculate the gravitational potential energy in terms of the confinement parameter of the electric field. We find that the extra dimensions are capable of amplifying the gravitational potential energy of the ion in a Rydberg state even when the compactification radius is smaller than the Bohr radius. This a consequence of the behavior of the potential $\chi_{s}$.

It is interesting that the short-distance behavior of the Green function associated to the gravitational potential plays an important role in the present situation. At the first sight, we could be led to think that the short-distance behavior is irrelevant when the ion is in the Rydberg state, since the probability to find the test particle close to the nucleus is very small in this state. Indeed this is the reason why the potential $\varphi_{s}$ is weak here. However, the source of the potential $\chi$ is spread in the space. Therefore, around any external point $\vec{x}$, there is an interval smaller than $R$, in the electrovacuum, where the short-distance Green function is dominant. It is through this term that the gravitational influence of the electrovacuum surrounding the test particle can increase significantly the gravitational potential energy of the ion.

Acknowledgements We thank the referee for useful comments which have helped us to improve this work. A. S. Lemos and E. Maciel thanks CAPES for financial support.
Open Access This article is distributed under the terms of the Creative Commons Attribution 4.0 International License (http://creativecomm ons.org/licenses/by/4.0/), which permits unrestricted use, distribution, and reproduction in any medium, provided you give appropriate credit to the original author(s) and the source, provide a link to the Creative Commons license, and indicate if changes were made.

Funded by $\mathrm{SCOAP}^{3}$.

\section{References}

1. N. Arkani-Hamed, S. Dimopoulos, G.R. Dvali, Phys. Lett. B 429, 263 (1998)

2. I. Antoniadis, N. Arkani-Hamed, S. Dimopoulos, G.R. Dvali, Phys. Lett. B 436, 257 (1998)

3. L. Randall, R. Sundrum, Phys. Rev. Lett. 83, 3370 (1999)

4. L. Randall, R. Sundrum, Phys. Rev. Lett. 83, 4690 (1999)

5. D.J. Kapner, T.S. Cook, E.G. Adelberger, J.H. Gundlach, B.R. Heckel, C.D. Hoyle, H.E. Swanson, Phys. Rev. Lett. 98, 021101 (2007)

6. J. Murata, S. Tanaka, Class. Quant. Gravit. 32, 033001 (2015)

7. S. Cullen, M. Perelstein, Phys. Rev. Lett. 83, 268 (1999)

8. S. Hannestad, G.G. Raffelt, Phys. Rev. D 67, 125008 (2003), Erratum-ibid.D, 69, 029901 (2004)

9. E.G. Giudice, R. Rattazzi, J.D. Wells, Nucl. Phys. B 544, 3-38 (1999)

10. G. Aad et al., Phys. Rev. Lett. 110, 011802 (2013)

11. G. Aad et al. CMS Collab., Phys. Lett. B 755, 102 (2016)

12. G. Aad et al., CMS Collab, Eur. Phys. J. C 75, 235 (2015)

13. G. Landsberg, Mod. Phys. Lett. A 50, 1540017 (2015)

14. K.A. Olive et al. (Particle Data Group), Chin. Phys. C, 38, 090001 (2014). (Extra dimensions, Updated September 2015 by John Parsons and Alex Pomarol)

15. F. Luo, H. Liu, Chin. Phys. Lett. 23, 2903 (2006)

16. F. Luo, H. Liu, Int. J. Theor. Phys. 46, 606 (2007)

17. Z.-G. Li, N. W-T, A.P. Patón, Chin. Phys. B 17, 70 (2008)

18. Z. Li, X. Chen, arXiv:1303.5146 [hep-ph]

19. L.B. Wang, W.T. Ni, Mod. Phys. Lett. A 28, 1350094 (2013)

20. Z. Wan-Ping, Z. Peng, Q. Hao-Xue, Open Phys. 13, 96 (2015)

21. E.J. Salumbides et al., New J. Phys. 17, 033015 (2015)

22. F. Dahia, A.S. Lemos, Phys. Rev. D 94(8), 084033 (2016)

23. U.D. Jentschura, P.J. Mohr, J.N. Tan, B.J. Wundt, Phys. Rev. Lett. 100, 160404 (2008)

24. P.J. Mohr, B.N. Taylor, D.B. Newell, Rev. Mod. Phys. 84, 1527 (2012)

25. T.W. Hänsch, Rev. Mod. Phys. 78, 1297 (2006)

26. L.-S. Ma et al., Science 303, 1843 (2004)

27. R. Pohl et al., Nature 466, 213 (2010)

28. A. Antognini et al., Science 339, 417 (2013)

29. C.E. Carlson, Prog. Part. Nucl. Phys. 82, 59 (2015)

30. J.J. Krauth et al., arXiv: 1706.00696

31. F. del Aguila, M. Perez-Victoria, J. Santiago, JHEP 0610, 056 (2006)

32. E.G. Adelberger, B.R. Heckel, S. Hoedl, C.D. Hoyle, D.J. Kapner, A. Upadhye, Phys. Rev. Lett. 98, 131104 (2007)

33. A. Kehagias, K. Sfetsos, Phys. Lett. B 472, 39 (2000)

34. V. Rubakov, M. Shaposhnikov, Phys. Lett. B 125, 136 (1983)

35. N. Arkani-Hamed, S. Dimopoulos, J. March-Russell, Phys. Rev. D 63, 064020 (2001)

36. I. Antoniadis, K. Benakli, A. Laugier, T. Maillard, Nucl. Phys. B 662, 40 (2003)

37. W.D. Goldberger, M.B. Wise, Phys. Rev. Lett. 83, 4922 (1999)

38. Z. Chacko, E. Perazzi, Phys. Rev. D 68, 115002 (2003)

39. E.G. Adelberger, B.R. Heckel, A.E. Nelson, Annu. Rev. Nucl. Part. Sci. 53, 77 (2003) 
40. S.B. Giddings, S. Thomas, Phys. Rev. D 65, 056010 (2002)

41. S. Dimopoulos, G. Landsberg, Phys. Rev. Lett. 87, 161602 (2001)

42. P.C. Argyres, S. Dimopoulos, J. March-Russell, Phys. Lett. B 441, $96(1998)$

43. D.B. Kaplan, Phys. Lett. B 288, 342 (1992)

44. S. Randjbar-Daemi, M. Shaposhnikov, Phys. Lett. B 492, 361364(2000)
45. N. Arkani-Hamed, M. Schmaltz, Phys. Rev. D 61, 033005 (2000)

46. R. Sundrum, Phys. Rev. D 59, 085009 (1999)

47. J.H. Noble, U.D. Jentschura, Phys. Rev. A 93, 032108 (2016)

48. F. Dahia, A.S. Lemos, Eur. Phys. J. C 76(8), 435 (2016) 